\title{
Decreased expression of thioredoxin interacting protein mRNA in inflamed colonic mucosa in patients with ulcerative colitis
}

\author{
YASUO TAKAHASHI ${ }^{1}$, HIDEKI MASUDA ${ }^{2}$, YUKIMOTO ISHII ${ }^{2}$, YAYOI NISHIDA ${ }^{1}$, \\ MEGUMI KOBAYASHI ${ }^{3}$ and SATOSHI ASAI ${ }^{1}$ \\ ${ }^{1}$ Division of Genomic Epidemiology and Clinical Trials, Advanced Medical Research Center, \\ Departments of ${ }^{2}$ Surgery and ${ }^{3}$ Pharmacology, Nihon University School of Medicine, \\ 30-1 Oyaguchi-Kami Machi, Itabashi, Tokyo 173-8610, Japan
}

Received February 6, 2007; Accepted March 19, 2007

\begin{abstract}
Ulcerative colitis (UC) is a chronic inflammatory bowel disease associated with intestinal inflammation and tissue damage. It is assumed that the oxidative stress that accompanies chronic inflammation contributes to the development of colorectal cancer (CRC). Thioredoxin interacting protein (TXNIP), whose expression is induced by various types of stress including oxidative stress and is downregulated in various types of human cancer including colorectal cancer, is a negative regulator of thioredoxin, which is an important regulator of redox balance. However, TXNIP expression in clinical specimens of UC remains unclear. Herein, using TaqMan RT-PCR assay, we demonstrated that TXNIP expression in UC and CRC colonic mucosa specimens was significantly lower than that in normal tissues $(\mathrm{p}=0.0007$ and $\mathrm{p}<0.0001$, respectively). In addition, in situ hybridization study showed that inflammatory cells expressing the TXNIP transcript were abundant in lymphoid follicles, the lamina propria and submucosa in the colonic mucosa of UC patients, but not in epithelial cells on the flat surface, whereas the TXNIP transcript was abundant in normal mucosa. Our results suggest that downregulation of TXNIP may be partly involved in the pathogenesis of UC, including inflammation and colitisassociated colon carcinogenesis.
\end{abstract}

\section{Introduction}

Patients with chronic inflammatory bowel disease (IBD) including ulcerative colitis (UC) are at an increased risk of

Correspondence to: Dr Satoshi Asai, Division of Genomic Epidemiology and Clinical Trials, Advanced Medical Research Center, Nihon University School of Medicine, 30-1 OyaguchiKami Machi, Itabashi, Tokyo 173-8610, Japan

E-mail: satoshi@med.nihon-u.ac.jp

Key words: thioredoxin interacting protein, vitamin D3 upregulated protein 1, ulcerative colitis, inflammatory bowel disease, colon cancer, in situ hybridization, TaqMan RT-PCR developing colorectal cancer (CRC). Various evidence implicates chronic inflammation as a key factor predisposing to CRC $(1,2)$. Oxidative stress induced by reactive oxygen and nitrogen species generated by inflammatory cells is thought to play a role in colon carcinogenesis in IBD, via the interaction with key genes involved in carcinogenic pathways such as p53 and DNA mismatch repair genes (3-5). However, it is not yet clear what gene(s) or factor(s) induced by oxidative stress are involved in the inflammatory and carcinogenic process in the inflamed colonic mucosa of UC patients.

Thioredoxin interacting protein (TXNIP), also known as VDUP1 (vitamin D3 up-regulated protein 1), is a negative regulator of thioredoxin (TRX) (6), an important regulator of redox balance $(7,8)$. TXNIP functions as an oxidative stress mediator inducing apoptosis by inhibiting TRX activity via the interaction between TRX and proliferation-associated gene (PAG) or between TRX and apoptosis signal-regulating kinase 1 (ASK-1) (9). TXNIP is induced by various types of stress, including heat shock, UV, $\gamma$-rays, anti-cancer agents and hydrogen peroxide $(9,10)$, which are well known to act as a common mediator of apoptosis in many types of cells. Moreover, TXNIP is related to tumorigenesis and its expression is reduced in various tumor tissues, including breast, lung and colorectal cancers $(11,12)$. Previously, we reported that TXNIP expression is significantly decreased in tumor tissue compared with normal tissue in colorectal and gastric cancer patients and is associated with clinical stage (13). We also reported that the TXNIP transcript is abundant in terminal differentiated cells in the colonic epithelium and in lymphocytes in the lymphoid follicles (14). It would be of interest to know whether TXNIP expression in the inflamed colonic mucosa of UC patients is increased by the oxidative stress that accompanies chronic inflammation, or whether it is decreased, the same as in sporadic colorectal cancers.

In this study, we investigated TXNIP expression in surgical specimens of inflamed colonic mucosa from $7 \mathrm{UC}$ patients and in tumor and adjacent normal colon specimens from 10 CRC patients, using TaqMan RT-PCR assay. Additionally, we examined the localization of TXNIP mRNA expression in inflamed colonic mucosa of UC patients using in situ hybridization. 
Table I. Analysis of covariance of TXNIP expression level in three groups of colon tissue.

\begin{tabular}{lccccc}
\hline Source & DF & Type III sum of squares & Mean square & F-value & $\mathrm{p}$ \\
\hline Group & 2 & 676.800 & 338.400 & 75.51 & $<.0001$ \\
Sex & 1 & 1.124 & 1.124 & 0.25 & 0.6214 \\
Age & 1 & 0.453 & 0.453 & 0.10 & 0.7534 \\
\hline
\end{tabular}

DF, degrees of freedom.

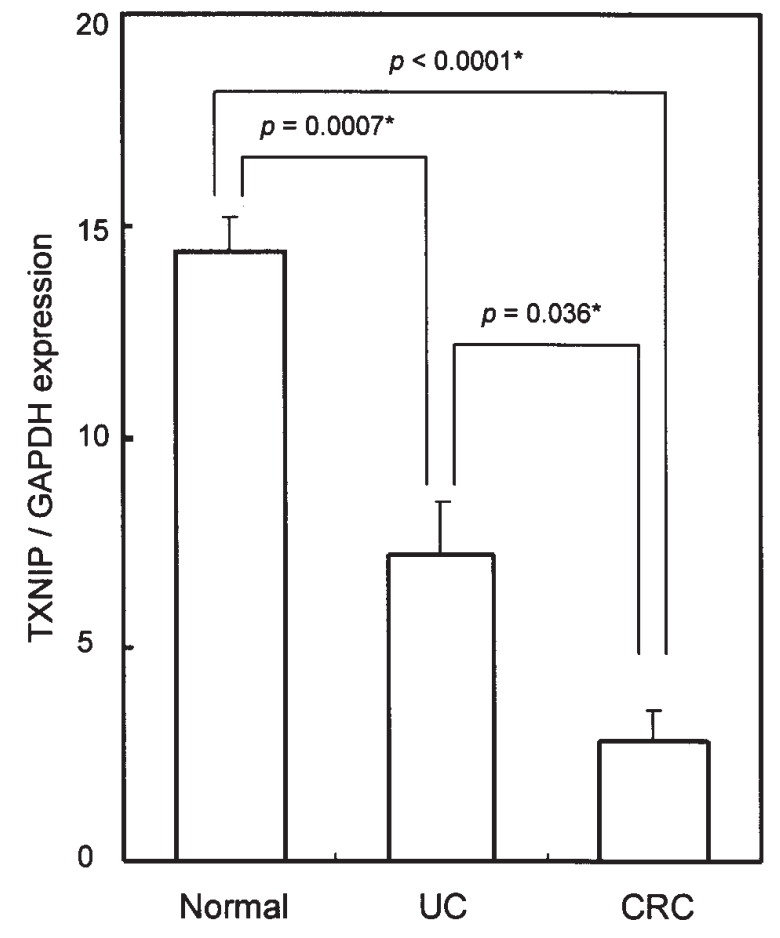

Figure 1. TXNIP expression in colonic mucosa of UC, CRC and normal groups. Data represent mean \pm SE of normal $(n=10), U C(n=7)$ and CRC groups $(\mathrm{n}=10)$. TXNIP expression in UC and CRC specimens was significantly lower than that in normal controls $(\mathrm{p}=0.0007$ and $\mathrm{p}<0.0001$, respectively).*: Tukey's multiple-comparison test.

\section{Materials and methods}

Sample collection. Seven UC patients who underwent total colectomy because of severe intractable colitis were included in this study; 4 men and 3 women with a median age of 36.1 years and age range of 20-58 years (UC group, $n=7$ ). Classical clinical, radiologic, endoscopic and histopathological criteria were used to confirm all diagnoses and all patients had comparable severe clinical disease (15). In addition, this study included 10 patients with colorectal cancer (CRC) with stage II disease; 6 men and 4 women with a median age of 63.9 years and age range of 39-71 years (CRC group, $n=10$ ). A portion of each specimen was used for routine histopathological examination. Normal control samples were obtained at least $5 \mathrm{~cm}$ from the colorectal cancer and were confirmed to be normal on histopathological assessment (Normal group, $\mathrm{n}=10$ ). All samples were washed in phosphate buffered saline to avoid contamination with blood and necrotic cells and were immediately frozen in liquid nitrogen and stored at $-80^{\circ} \mathrm{C}$ until further use. A portion of each specimen was immediately embedded in OCT compound in hexane and dry ice and stored at $-80^{\circ} \mathrm{C}$ until sectioning.

TaqMan RT-PCR assay. To examine TXNIP gene expression, TaqMan RT-PCR assay was performed using an ABI Prism 7700 Sequence Analyzer (PE Applied Biosystems, Foster City, CA) as described previously (16). In brief, total RNA was isolated using RNeasy (Qiagen Inc., Chatsworth, CA), and DNase treatment was performed using a Message Clean Kit (GenHunter Corp., Brookline, MA), according to the manufacturer's instructions. One-step RT-PCR of $30 \mathrm{ng}$ total RNA was carried out in a $50-\mu 1$ reaction under the following conditions: $2 \mathrm{~min}$ at $50^{\circ} \mathrm{C}, 30 \mathrm{~min}$ at $60^{\circ} \mathrm{C}, 5 \mathrm{~min}$ at $95^{\circ} \mathrm{C}$ and then 40 cycles of amplification for $20 \mathrm{sec}$ at $95^{\circ} \mathrm{C}$ and $1 \mathrm{~min}$ at $60^{\circ} \mathrm{C}$. Triplicate PCR amplifications were carried out for each sample. Primers and the TaqMan probe for glyceraldehyde-3phosphate dehydrogenase (GAPDH) and TXNIP as described previously were used (10). The Universal Human Reference RNA (Stratagene, La Jolla, CA) was used as control RNA to generate each standard curve for the relative concentration of TXNIP or GAPDH. The normalized concentration of TXNIP was determined by dividing the concentration of TXNIP by the concentration of GAPDH. Analysis of covariance (ANCOVA) was used to evaluate the relationship between TXNIP expression and three covariates i.e., sex, age and types of tissues (tissue group). A multiple-comparison test (TukeyKramer HSD post-hoc analysis) was used to analyze the differences in means of TXNIP expression among the three groups of UC, CRC and normal colon tissue. A result was considered statistically significant at $\mathrm{p}<0.05$. The statistical analysis was performed with SAS 9.1.3 (SAS Institute Inc., Cary, NC) statistical software.

In situ hybridization (ISH) analysis. Tissue samples (2 from UC and 3 from normal colon) were subjected to ISH analysis. The detailed protocol of this assay was described previously (14). Briefly, $10-\mu \mathrm{m}$-thick sections were cut $\left(-15^{\circ} \mathrm{C}\right.$, HM560M, MICROM, Walldorf, Germany) and mounted on silanized slides (Matsunami, Osaka, Japan). All sections were fixed in $4 \%$ paraformaldehyde-PBS for $15 \mathrm{~min}$, incubated twice in PBS containing $0.1 \%$ active DEPC for $15 \mathrm{~min}$ and equilibrated in 5X SSC for $15 \mathrm{~min}$. Hybridization was performed in a hybridization buffer $(50 \%$ formamide, $5 \mathrm{X}$ SSC, $5 \mathrm{X}$ Denhardt's solution, $500 \mu \mathrm{g} / \mathrm{ml}$ salmon sperm DNA, $250 \mu \mathrm{g} / \mathrm{ml} \mathrm{t}-\mathrm{RNA}, 1 \mathrm{mM}$ DTT) containing antisense or sense riboprobes at $500 \mathrm{ng} / \mathrm{ml}$ in a humid chamber for $20 \mathrm{~h}$ 


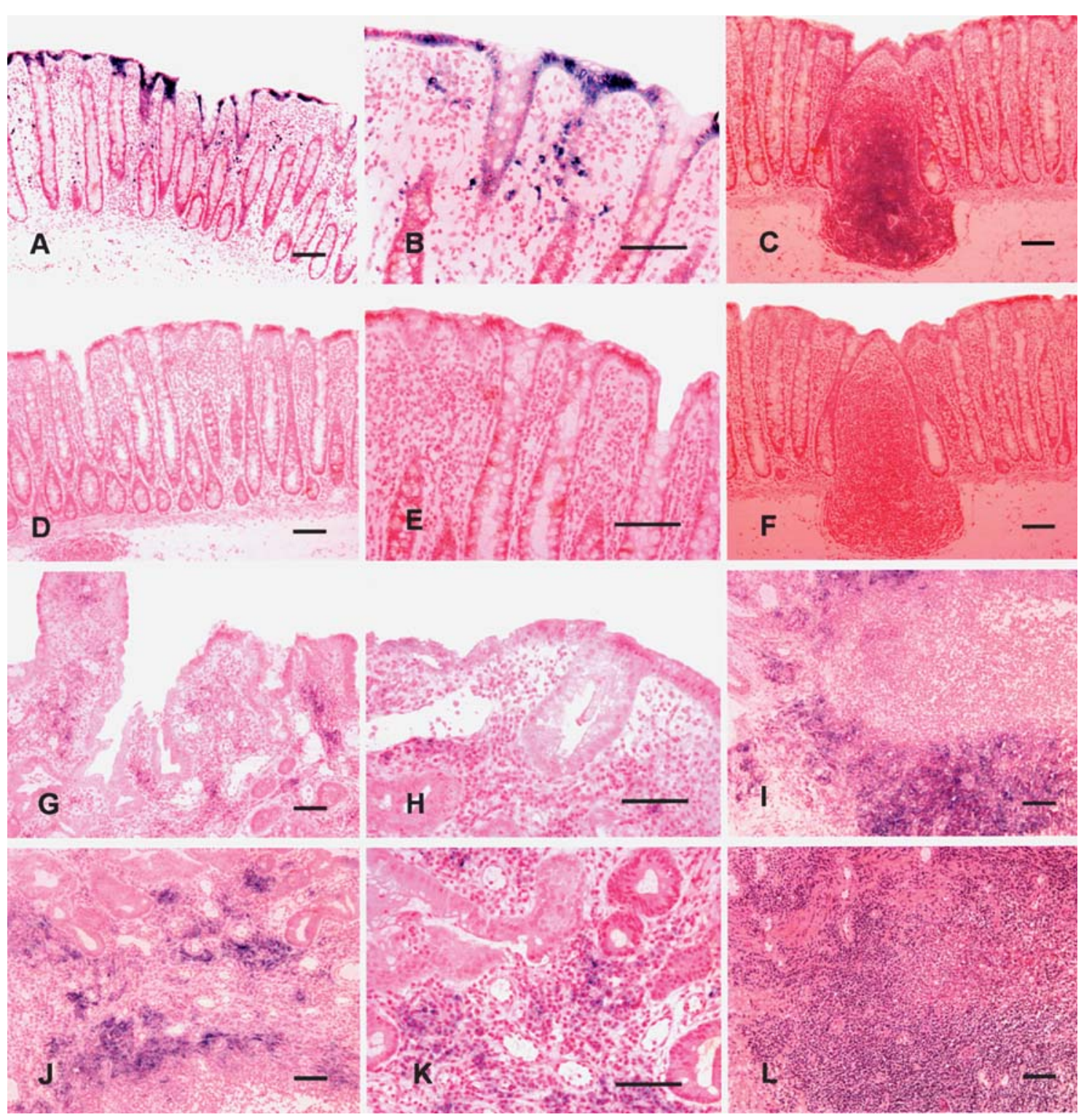

Figure 2. Localization of TXNIP mRNA in inflamed colonic mucosa of UC patients (G-L) and normal control (A-F). Antisense hybridization (A, B, C, G, H, I, J, K); sense hybridization (D-F); HE staining (L). TXNIP transcript is abundant on the flat surface of the colonic crypts (A, B) and in a lymphoid follicle (C) in normal colon tissue. TXNIP mRNA signal is observed in superficial epithelial cells on the flat surface (B). Cells expressing the TXNIP transcript were sparse in the lamina propria of the colonic epithelium in normal tissue (B), but were abundant in the inflamed mucosa of UC patients (G-K). TXNIP-positive cells were observed in the submucosa of UC samples (I-K). The epithelial cells were partially exfoliated and TXNIP-positive epithelial cells were sparse in the colonic mucosa of UC patients (G, H). Sense probe hybridization produced no signal over background (D-F). Bar, $100 \mu \mathrm{m}$.

at $58^{\circ} \mathrm{C}$. After hybridization, the sections were washed in $2 \mathrm{X}$ SCC for $30 \mathrm{~min}$ at room temperature (RT), then in $2 \mathrm{X}$ SCC and $0.1 \mathrm{X} \mathrm{SCC}$ for $1 \mathrm{~h}$ at $65^{\circ} \mathrm{C}$ and finally incubated in a TBST buffer (125 mM Tris, $150 \mathrm{mM} \mathrm{NaCl}, 2 \mathrm{mM} \mathrm{KCl,} 0.1 \%$ Tween-20, pH 8.0) for $15 \mathrm{~min}$ at RT. For digoxigenin detection, the sections were blocked with Dako Protein Block Serum-Free (Dako, Kyoto, Japan) for $20 \mathrm{~min}$ and then incubated with alkaline phosphatase-conjugated antidigoxgenin antibody (Roche Diagnostics, Basel, Switzerland) diluted 1:500 in a blocking buffer (Roche Diagnostics) for $1 \mathrm{~h}$ at RT. After washing in TBST with $2 \mathrm{mM}$ levamisole, the signal was visualized by overnight incubation with BM purple substrate (Roche Diagnostics). Finally, the sections were counterstained with nuclear fast red and mounted in NEW MX (MATSUNAMI). Digoxigenin (DIG)-labeled riboprobes (sense and antisense) for TXNIP (424 bp, nucleotides 2046-2470 of the TXNIP mRNA sequence, GenBank XM_002093) as described previously were used (14).

\section{Results}

The expression of TXNIP mRNA was examined in specimens of inflamed colonic mucosa from 7 UC patients and in specimens of tumor and adjacent normal colon from 10 CRC patients, using TaqMan RT-PCR assay. The results of analysis of covariance are shown in Table I. The effect of the group was significant, but for those of sex and age were not. Tukey's multiple-comparison test showed that the expression level of TXNIP mRNA was significantly different between the normal 
group and each of the other two groups (Fig. 1). The level of TXNIP mRNA in the UC and CRC groups was $7.23 \pm 1.23$ (mean $\pm \mathrm{SE}$ ) and $2.82 \pm 0.75$ (mean $\pm \mathrm{SE}$ ), respectively, while that in the normal group was $14.43 \pm 0.75$ (mean \pm SE). TXNIP expression in UC and CRC specimens was significantly lower than that in normal tissue $(\mathrm{p}=0.0007$ and $\mathrm{p}<0.0001$, respectively). The difference in TXNIP expression between the UC and CRC specimens was also significant $(\mathrm{p}=0.036)$.

ISH analysis revealed that the TXNIP transcript was abundant in epithelial cells in the upper portion of the colonic crypts, especially on the flat surface and in lymphocytes in lymphoid follicles in normal colon (Fig. 2A-C). Also, sparse cells expressing the TXNIP transcript were present in the lamina propria of the colonic epithelium (Fig. 2A and 2B). On the contrary, inflammatory cells expressing the TXNIP transcript were abundant in the lamina propria and submucosa of the colonic mucosa of UC patients, as a result of a concomitant increase in infiltrating inflammatory cells (Fig. 2G-L). Epithelial cells expressing the TXNIP transcript at the luminal surface were obviously decreased in the colonic mucosa of UC patients compared to normal controls (Fig. 2G and 2H), though parts of the mucosal epithelium were exfoliated due to severe inflammation.

\section{Discussion}

In this study, TaqMan RT-PCR assay revealed that the level of TXNIP mRNA was significantly decreased in the colonic mucosa of UC patients compared to normal controls, although the difference was only 2-fold. Additionally, the ISH study revealed that inflammatory cells expressing the TXNIP transcript were increased in the lamina propria and submucosa of UC samples compared to normal controls. Nevertheless, colonic epithelial cells expressing the TXNIP transcript were decreased in UC samples. These discrepancies between inflammatory cells and epithelial cells may be due to the following reasons. First, all of the UC specimens used in this study were obtained from patients with severe intractable colitis, leading to damage or exfoliation of epithelial cells. In these specimens, TXNIP expression in epithelial cells may be markedly affected by cell damage, but not by oxidative stress. Second, continuous oxidative stress that accompanies chronic inflammation may have a different influence on TXNIP expression in epithelial cells from temporary oxidative stress, which has been reported to induce the TXNIP gene in vitro. Third, the immune reaction associated with the proliferation of inflammatory cells in actively inflamed colonic mucosa may have been the consequence of an increase in infiltrating cells expressing the TXNIP transcript in the lamina propria and submucosa of UC samples. Our TaqMan RT-PCR and ISH study suggested that the decreased expression of TXNIP mRNA in UC specimens, despite the increase in inflammatory cells expressing the TXNIP transcript, may have been caused by the loss or decrease of TXNIP expression in epithelial cells in colonic mucosa with severe colitis.

Various clinical evidence has implicated TXNIP as a novel tumor and metastasis suppressor, of which expression is dramatically reduced in various tumor tissue, including breast, lung and colorectal cancer (CRC) (11-13). Retroviral transfection of TXNIP to human promyelocytic leukemia
HL-60 cells suppresses cell growth, supporting that TXNIP has a tumor-suppressive effect (11). In this study, TXNIP expression was significantly decreased (5.1-fold difference) in tumor tissue of CRC patients compared to normal controls, consistent with previous studies. Also, we demonstrated that TXNIP expression was significantly decreased in the inflamed colonic mucosa of UC patients compared to normal controls. Because many of the molecular alterations responsible for sporadic CRC development also play a role in colitisassociated colon carcinogenesis, the mechanisms of the reduction of TXNIP expression in epithelial cells may be common between UC and CRC, e.g. DNA-methylation (17). It is still controversial whether TXNIP is involved in carcinogenesis in inflamed colonic mucosa of UC patients or whether it is involved in the inflammatory process, because all UC specimens showed severe inflammation. However, the possibility that the loss or decrease of the tumor-suppressor function of TXNIP may lead to an increased risk of developing CRC in UC patients cannot be ruled out, although this study showed only a 2-fold decrease in TXNIP expression in UC samples compared to normal controls. In future clinical studies, we will compare TXNIP expression between inactive and active colonic inflammation to examine the relationship between the degree of inflammation and TXNIP expression in the colorectal mucosa of UC patients.

Previous analysis demonstrated that TXNIP inhibited thioredoxin (TRX), which maintains the cellular redox state (7), suggesting that the TRX-TXNIP interaction may also be important as a redox regulatory mechanism in cellular processes. Our study demonstrated that the expression of TXNIP mRNA was decreased in the inflamed colonic mucosa of UC patients despite the increase in inflammatory cells expressing the TXNIP transcript. These observations suggest that redox regulation may be involved in the carcinogenic and inflammatory processes in the colonic mucosa of UC patients. Our study provides new insights into the molecular mechanisms underlying carcinogenesis in UC patients via chronic inflammation.

\section{Acknowledgements}

This study was supported in part by a Grant from the Japanese Ministry of Education, Culture, Sports, Science and Technology to promote advanced scientific research, awarded to Nihon University, and by a Grant-in-Aid from the Ministry of Education, Science, Sports and Culture of Japan (no. 16591359).

\section{References}

1. Itzkowitz SH: Cancer prevention in patients with inflammatory bowel disease. Gastroenterol Clin North Am 31: 1133-1144, 2002.

2. Itzkowitz SH and Yio X: Inflammation and cancer IV. Colorectal cancer in inflammatory bowel disease: the role of inflammation. Am J Physiol Gastrointest Liver Physiol 287: G7-G17, 2004.

3. Brentnall TA, Crispin DA, Rabinovitch PS, et al: Mutations in the p53 gene: an early marker of neoplastic progression in ulcerative colitis. Gastroenterology 107: 369-378, 1994.

4. Brentnall TA, Crispin DA, Bronner MP, et al: Microsatellite instability in nonneoplastic mucosa from patients with chronic ulcerative colitis. Cancer Res 56: 1237-1240, 1996.

5. Hofseth LJ, Saito S, Hussain SP, et al: Nitric oxide-induced cellular stress and p53 activation in chronic inflammation. Proc Natl Acad Sci USA 100: 143-148, 2003. 
6. Nishiyama A, Matsui M, Iwata S, et al: Identification of thioredoxin-binding protein-2/vitamin $\mathrm{D}$ (3) up-regulated protein 1 as a negative regulator of thioredoxin function and expression. $\mathrm{J}$ Biol Chem 274: 21645-21650, 1999.

7. Yodoi J, Nakamura $\mathrm{H}$ and Masutani $\mathrm{H}$ : Redox regulation of stress signals: possible roles of dendritic stellate TRX producer cells (DST cell types). Biol Chem 383: 585-590, 2002.

8. Chung JW, Jeon JH, Yoon SR and Choi I: Vitamin D3 upregulated protein 1 (VDUP1) is a regulator for redox signaling and stress-mediated diseases. J Dermatol 33: 662-669, 2006.

9. Junn E, Han SH, Im JY, et al: Vitamin D3 up-regulated protein 1 mediates oxidative stress via suppressing the thioredoxin function. J Immunol 164: 6287-6295, 2000.

10. Takahashi Y, Nagata T, Ishii Y, Ikarashi M, Ishikawa K and Asai S: Up-regulation of vitamin D3 up-regulated protein 1 gene in response to 5-fluorouracil in colon carcinoma SW620. Oncol Rep 9: 75-79, 2002.

11. Han SH, Jeon JH, Ju HR, et al: VDUP1 up-regulated by TGFbeta1 and 1,25-dihydroxyvitamin D3 inhibits tumor cell growth by blocking cell-cycle progression. Oncogene 22: 4035-4046, 2003.
12. Butler LM, Zhou X, Xu WS, et al: The histone deacetylase inhibitor SAHA arrests cancer cell growth, up-regulates thioredoxin-binding protein-2 and down-regulates thioredoxin. Proc Natl Acad Sci USA 99: 11700-11705, 2002.

13. Ikarashi M, Takahashi $Y$, Nagata T, Ishii $Y$, Ishikawa $K$ and Asai S: Vitamin D3 up-regulated protein 1 (VDUP1) expression in gastrointestinal cancer and its relation to stage of disease. Anticancer Res 22: 4045-4048, 2002.

14. Takahashi Y, Ishii Y, Murata A, Nagata T and Asai S: Localization of thioredoxin-interacting protein (TXNIP) mRNA in epithelium of human gastrointestinal tract. J Histochem Cytochem 51: 973-976, 2003.

15. Truelove SC and Witts LJ: Cortisone in ulcerative colitis; final report on a therapeutic trial. Br Med J 2: 1041-1048, 1955.

16. Kasahara M, Takahashi Y, Nagata T, et al: Thymidylate synthase expression correlates closely with E2F1 expression in colon cancer. Clin Cancer Res 6: 2707-2711, 2000.

17. Ahsan MK, Masutani H, Yamaguchi Y, et al: Loss of interleukin-2-dependency in HTLV-I-infected T cells on gene silencing of thioredoxin-binding protein-2. Oncogene 25: 2181$2191,2006$. 